\title{
Empirical Formulas for Performance Prediction of Concrete Embedded Antenna
}

\author{
$\mathrm{Ju} \operatorname{Tan}^{1}$, Yu Shao ${ }^{1 *}$, Jiliang Zhang ${ }^{1}$, and Jie Zhang ${ }^{1} 2$ \\ ${ }^{1}$ Department of Electronic and Electrical Engineering, The University of Sheffield, The United Kingdom \\ ${ }^{2}$ Ranplan Wireless Network Design Ltd., Cambridge, The United Kingdom \\ * Corresponding author, email: y.shao@sheffield.ac.uk
}

\begin{abstract}
To mitigate the space occupation and aesthetic problems of indoor dense small cell deployment, a microstrip antenna with multiple layer configuration operating at $3.5 \mathrm{GHz}$ is embedded in concrete all for indoor communication. The impact of embedding depth and concrete dielectric constant on antenna gain and input reactance are investigated, and simple empirical formulas are obtained based on full-wave simulation. The results in present work provide a good guidance to antenna designers and architects for concrete embedded antenna deployment and communication-friendly building materials selection.
\end{abstract}

Keywords-Concrete embedded antenna, empirical formula, performance prediction.

\section{INTRODUCTION}

Ultra dense small cell deployment is regarded as the most promising way to meet the traffic demand, and deploying small cells densely in buildings is anticipated to improve throughput in the next generation of cellular communication [1]. However, deployment of small cells with a number of antennas or antenna arrays will occupy extra space which leads to negative effects on usage of the buildings. A feasible solution to mitigate these negative effects is to embed antennas into the building materials. Most researches on concrete embedded antennas were focused on data and power transmission of wireless sensors embedded in concrete to monitor structural health [2]. In [3], a concrete floor embedded RFID tag antenna was presented. A feasible embedded antenna requires not only high electromagnetic performance but also robust mechanical behaviour. In [4], a structurally integrated antennas with multilayered structure have been proposed. To the best of our knowledge, no research on concrete embedded antenna for indoor communication has been reported.

In fact, the embedding is challenging since the strong coupling between antenna and concrete can significantly affect antenna performance. Thus, for obtaining the optimal antenna performance, the concrete electrical property should be taken into account in the building design stage. In this paper, a concrete embedded antenna is proposed and its gain and input reactance variations against embedding depth and concrete dielectric constant are investigated by full-wave simulation. Furthermore, simple empirical formulas are fitted based on simulation results to facilitate fast prediction of antenna performance.

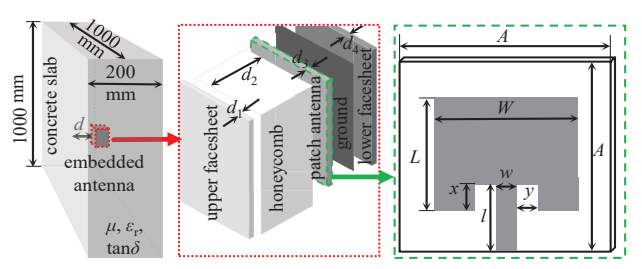

Fig. 1: Overview of antenna model geometry

TABLE I: Electrical properties and thickness for each layer

\begin{tabular}{ccccc}
\hline Layer & Material & $\epsilon_{r}$ & $\tan \delta$ & Thickness $(\mathrm{mm})$ \\
\hline UF & Roger 3003 & 3 & 0.001 & $d_{1}=0.25$ \\
Honeycomb & Air & 1 & 0 & $d_{2}=10$ \\
Substrate & Roger 5880 & 2.2 & 0.0009 & $d_{3}=1$ \\
LF & Roger 3003 & 3 & 0.001 & $d_{4}=0.25$ \\
\hline
\end{tabular}

TABLE II: Dimensional parameters of the proposed antenna

\begin{tabular}{lllccccc}
\hline Parameter & $A$ & $W$ & $L$ & $x$ & $y$ & $w$ & $l$ \\
\hline Value $(\mathrm{mm})$ & 60 & 33.85 & 28.39 & 8 & 2.69 & 3.12 & 23.805 \\
\hline
\end{tabular}

\section{SYSTEM MODEL}

In current work, the structurally integrated antenna proposed in [4] is selected due to its excellent mechanical and electrical performances. As shown in Fig. 1, the antenna is fully embedded in a solid concrete slab with a dimension of $1000 \mathrm{~mm} \times 1000 \mathrm{~mm} \times 200 \mathrm{~mm}$, the embedding depth $d$ is measured as the distance from the front concrete-air interface to the top surface of antenna. The concrete dielectric constant $\epsilon_{r}$ varies from 4 to 9 , and the loss tangent is fixed to be 0.03 $(\tan \delta=0.03)$. The multilayer configuration of the structurally integrated antenna is shown in Fig. 1. The antenna mainly consists of a microstrip patch etched on a Rogers RT5880 substrate and is sandwiched among a lower facesheet (LF), a honeycomb structure and an upper facesheet (UF). The electrical properties and thickness of each layer are listed in Table I. The patch is optimized to operate at $3.5 \mathrm{GHz}$, and its dimensions are shown in Table II. The impact of the embedding depth and the concrete dielectric constant on antenna will be investigated, so the antenna configuration and dimension are fixed. All the simulations are performed by Computer Simulation Technology (CST) Studio.

\section{RESULTS AND DISCUSSION}

Fig. 2 (a) and (b) show the variations of gain $G$ and $X_{\text {in }}$ against the embedding depth and the dielectric constant. It is 
noted that the increase of $d$ makes $G$ decrease in a fluctuation, while the increasing $\epsilon_{\mathrm{r}}$ leads to the decline of $G$. Thus, the gain $G$ can be formulated as a linear decreasing function superposed with a damping sinusoidal function as (1). The input reactance oscillates damply with the embedding depth, so it can be modeled by a sinusoidal function with attenuated amplitude as (2):

$$
\begin{aligned}
& G=A_{1}\left(\epsilon_{\mathrm{r}}\right) e^{B_{1}\left(\epsilon_{\mathrm{r}}\right) d} \sin \left[C_{1}\left(\epsilon_{\mathrm{r}}\right) d\right]+D_{1}\left(\epsilon_{\mathrm{r}}\right)\left[d+E_{1}\left(\epsilon_{\mathrm{r}}\right)\right], \\
& X_{\mathrm{in}}=A_{2}\left(\epsilon_{\mathrm{r}}\right)+B_{2}\left(\epsilon_{\mathrm{r}}\right) e^{C_{2}\left(\epsilon_{\mathrm{r}}\right) d} \cos \left(D_{2}\left(\epsilon_{\mathrm{r}}\right) d+E_{2}\left(\epsilon_{\mathrm{r}}\right)\right),
\end{aligned}
$$

where $G$ and $X_{\text {in }}$ are measured in $\mathrm{dBi}$ and $\mathrm{Ohm}$, respectively, and $d$ is measured in meter.

In formula (1) and (2), $A_{i}, B_{i}, C_{i}, D_{i}$ and $E_{i}(i=1,2)$ are undetermined coefficients which are functions of $\epsilon_{\mathrm{r}}$. By nonlinear fitting, formulas of $G$ for some dielectric constants are listed in Table III. After careful inspection of the results in Table III, the coefficients in formula (1) can be formulated as linear function of $\epsilon_{\mathrm{r}}$, and the fitted formulas for the five coefficients in (1) are listed in Table IV. Substituting the results in Table IV into (1), the empirical formula of $G$ as a function of $d$ and $\epsilon_{\mathrm{r}}$ can be written as:

$$
\begin{aligned}
G & =\left(0.53 \epsilon_{\mathrm{r}}-0.89\right) e^{\left(1.47 \epsilon_{\mathrm{r}}-32.75\right) d} \sin \left[\left(22.09 \epsilon_{\mathrm{r}}+250.80\right) d\right] \\
& +\left(-1.43 \epsilon_{\mathrm{r}}-9.98\right)\left[d+\left(0.05 \epsilon_{\mathrm{r}}-0.54\right)\right] .
\end{aligned}
$$

Following the same fitting procedure of $G$, the formulas of $X_{\text {in }}$ as a function of $d$ and $\epsilon_{\mathrm{r}}$ can be written as:

$$
\begin{aligned}
X_{\text {in }} & =\left(0.76 \epsilon_{\mathrm{r}}-6.16\right)+\left(0.65 \epsilon_{\mathrm{r}}+10.52\right) e^{\left(-0.36 \epsilon_{\mathrm{r}}-17.94\right) d} \\
& \times \cos \left[\left(29.35 \epsilon_{\mathrm{r}}+175.50\right) d+\left(-0.02 \epsilon_{\mathrm{r}}+8.13\right)\right] .
\end{aligned}
$$

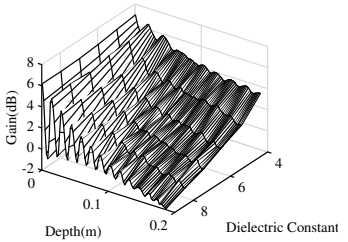

(a) $G$ against $d$ and $\epsilon_{\mathrm{r}}$

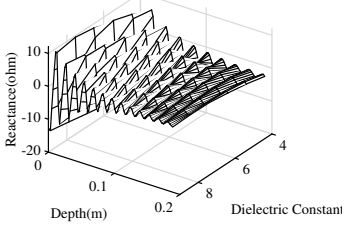

(b) $X_{\text {in }}$ against $d$ and $\epsilon_{\mathrm{r}}$
Fig. 2: Simulated results.

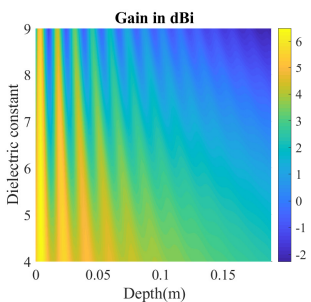

(a) $G$ against $d$ and $\epsilon_{\mathrm{r}}$

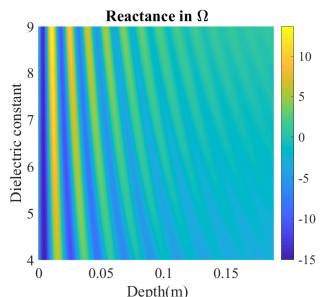

(b) $X_{\text {in }}$ against $d$ and $\epsilon_{\mathrm{r}}$
Fig. 3: Fitted results.

Fig. 3 (a) presents the fitted result of $G$, the gain of proposed antenna decrease in a fluctuating manner with the
TABLE III: Formulas of gain for different $\epsilon_{r}$

\begin{tabular}{cc}
\hline$\epsilon_{r}$ & Nonlinear regression formulation \\
\hline 4 & $G=0.93 e^{-26.28 d} \sin (342.50 d)-16.03 \cdot(d-0.37)$ \\
6 & $G=2.31 e^{-24.50 d} \sin (381.40 d)-18.88 \cdot(d-0.21)$ \\
8 & $G=3.31 e^{-20.97 d} \sin (429.10 d)-20.82 \cdot(d-0.14)$ \\
\hline
\end{tabular}

TABLE IV: Linear fitting formulas for coefficients in (1)

\begin{tabular}{cc}
\hline Coefficient & Formulation \\
\hline$A_{1}$ & $A_{1}=0.53 \epsilon_{\mathrm{r}}-0.89$ \\
$B_{1}$ & $B_{1}=1.47 \epsilon_{\mathrm{r}}-32.75$ \\
$C_{1}$ & $C_{1}=22.09 \epsilon_{\mathrm{r}}+250.80$ \\
$D_{1}$ & $D_{1}=-1.43 \epsilon_{\mathrm{r}}-9.98$ \\
$E_{1}$ & $E_{1}=0.05 \epsilon_{\mathrm{r}}-0.54$ \\
\hline
\end{tabular}

increasing of $d$, since a larger $d$ introduces more absorption loss by the concrete. The fluctuation is caused by the interference of multiple reflection inside the concrete slab. The local maximum of gain occurs once the multiple reflection are in phase. It is worth noting that the fluctuation period is approximately equal to half-wavelength in the concrete. The $X_{\text {in }}$ oscillates with gradually decaying amplitude as $d$ increases, and the fitted result is shown in Fig. 3 (b). The concrete slab can be modeled as a lossy transmission line, so the variation of $X_{\text {in }}$ against $d$ is similar to the reactance fluctuation along a transmission line.

\section{CONCLUSion}

A microstrip antenna is embedded into a concrete wall for indoor communication. How antenna performances are affected by both the embedding depth and the concrete dielectric constant was investigated. It is found that the gain decrease with the increase of embedding depth, and the input reactance displays a damped periodical oscillation with the embedding depth. It is also found that a larger concrete dielectric constant leads to smaller gain. Simple empirical formulas are fitted as two-dimensional regression function of the embedding depth and the dielectric constant for performance prediction, which facilitate the concrete embedded antenna deployment and communication-friendly building materials selection.

\section{ACKNOWLEDGMENT}

The research is funded in part by the European Union's H2020-MSCA-IF-AceLSAA (752644) and in part by the European Union's H2020-MSCA-IF-GATE (843133).

\section{REFERENCES}

[1] J. Zhang, A. A. Glazunov and J. Zhang, "Wireless energy efficiency evaluation for building under design based on analysis of interference gain," IEEE Trans. Veh. Tech., vol. 69, no. 6, pp. 6310-6324, 2020.

[2] G. Castorina, L. Di Donato, A. F. Morabito, T. Isernia and G. Sorbello, "Analysis and design of a concrete embedded antenna for wireless monitoring applications [antenna applications corner]," IEEE Antennas Propag. Mag., vol. 58, no. 6, pp. 76-93, 2016.

[3] K. M. Z. Shams and M. Ali, "Wireless power transmission to a buried sensor in concrete," IEEE Sensors J., vol. 7, no. 12, pp. 1573-1577, 2007.

[4] C. You, M. M. Tentzeris and W. Hwang, "Multilayer effects on microstrip antennas for their integration with mechanical structures," IEEE Trans. Antennas Propag., vol. 55, no. 4, pp. 1051-1058, 2007. 